\title{
ACE FlexGrid Telescope Flexure and Pointing Software
}

\author{
Peter Mack ${ }^{1}$, John Stein ${ }^{1,2}$, Wonyong $\operatorname{Han}^{3}$ \\ ${ }^{1}$ Astronomical Consultants \& Equipment, Inc., P.O. Box 91946, Tucson \\ AZ 85741, USA \\ ${ }^{2}$ Mathematics Department, Geneva College, Beaver Falls, PA 15010, \\ USA \\ ${ }^{3}$ Korea Astronomy Observatory, Taejon, 305-348, Korea
}

\begin{abstract}
We describe ACE FlexGrid, a telescope flexure and pointing model based on an empirical grid of reference points. This software is valid for all types of telescopes and is especially suited to robotic telescopes which repeatedly observe the same object.
\end{abstract}

\section{Introduction}

Some pointing models make assumptions about the type of mount and how it will flex at different positions on the sky. We have taken a different approach which assumes nothing about the type of telescope mount. The only assumption is that the telescope flexure is repeatable for a given load and that for significantly different loads a new map will be created.

\section{The Flex Grig Database}

Using one of the standard catalogs of bright stars with accurate positions and proper motions a set of grid points is created. These points can be randomly placed; they do not need to be in a regular grid pattern. Stars are also observed along the observing horizon to create a set of boundary points (Figure 1).

A database entry is made for each grid or boundary point. The catalog position of the star, the observed Hour Angle and Declination corrections, the temperature and barometric pressure are saved.

\section{Flex Grid Interpolation}

Once the flex grid has been generated the software makes interpolations between the target star and the reference points (Figure 2). Grid points are selected that "box" the target. Arc, triangular or trapezoidal boxing is created, in increasing order of preference, depending on the geometry. All interpolations are done along great circle arcs. Grid point mapping of the boundaries allows for ease in specifying unusual shapes. The telescope control software can monitor the telescope position and prevent it from crossing exclusion zone boundaries. It 


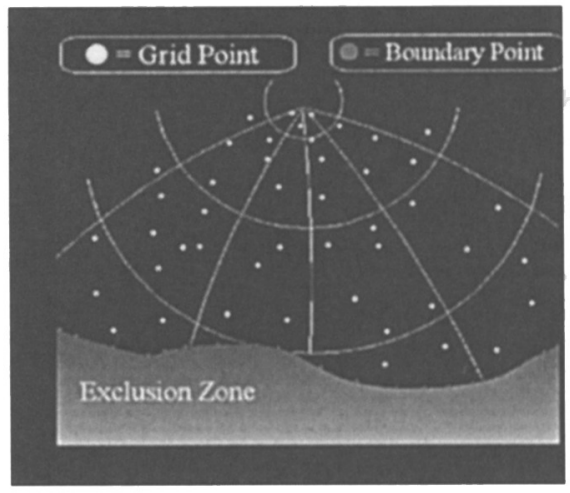

Figure 1. The Flex Grid

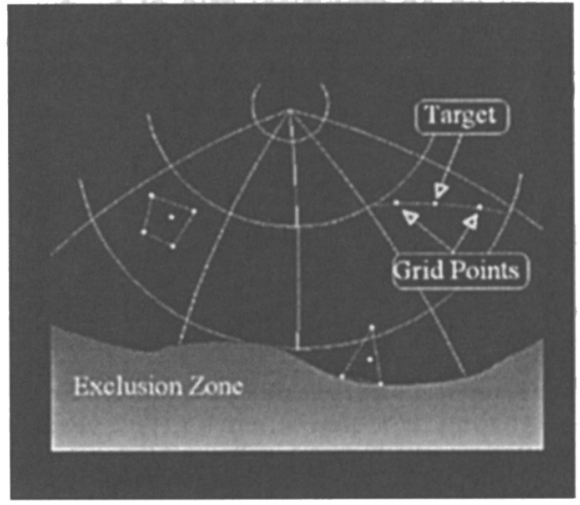

Figure 2. ACE FlexGrid interpolation

can also report the time before the telescope reaches a boundary, such as the observing horizon or interference with a support pier.

Each time an object is observed it can be added to the database if certain criteria are met (to prevent misidentified stars from being added to the database). Robotic telescopes tend to observe the same object on many nights. By saving the grid points every 10 minutes, an extensive set of grid points can be generated. The system is still to be tested but closure errors of only a few arc seconds are expected. The software is being used with the Korea Astronomy Observatory $1.0-\mathrm{m}$ Robotic Telescope described in these proceedings.

\section{Summary}

Pointing model routines suitable for robotic telescopes have been developed. Called the ACE FlexGrid, it is a part of the ACE Observatory Control System. It will also be made available as a library to integrate into other telescope control systems. More information is available at http://www.astronomical.com. 